\title{
Human Papillomavirus in Cervical Cancer
}

\author{
F. Xavier Bosch, MD and Silvia de Sanjosé, $M D$
}

\begin{abstract}
Address
Servei d’Epidemiologia i Registre del Càncer, Institut Català d'Oncologia, Av. Gran Via, s/n. Km 2.7, 08907 L'Hospitalet de Llobregat, Barcelona, Spain.

E-mail: x.bosch@ico.scs.es
\end{abstract}

Current Oncology Reports 2002, 4:175-183

Current Science Inc. ISSN I523-3790

Copyright (C) 2002 by Current Science Inc.

Epidemiologic studies supported by molecular technology have provided sufficient evidence of the causal role of some human papillomavirus (HPV) infections in the development of cervical cancer. The finding is consistent universally, and HPV has been proposed as the first identified necessary cause of cervical cancer. Such recognition translates into the concept that cervical cancer does not develop without persistent presence of HPV DNA. In the developed parts of the world, cytologic screening programs could benefit from the addition of HPV testing to their protocols. Controlled studies and one randomized trial have shown that HPV testing is helpful in solving the ambiguous cases generated by cytology reading. In populations where cytology programs are not functional or efficient, HPV testing is being evaluated as an alternative means of primary screening. Prevention of exposure to high-risk HPV types, either by prophylactic vaccination or by combined prophylactic and therapeutic immunologic intervention, may prove to be the most efficient and logistically feasible option for the prevention of cervical cancer in developing populations.

\section{Introduction}

A major discovery in human cancer etiology has been the recognition that cervical cancer is a rare consequence of persistent infection by some types of human papillomavirus (HPV). In public health terms, the importance of this finding is comparable with the unveiling of the association between cigarette smoking and lung cancer, or between persistent infections with hepatitis $B$ or hepatitis $C$ virus (HBV or HCV) and the risk of liver cancer. Moreover, as in the model of hepatitis B infection, intense efforts are currently being placed in the development and testing of vaccines that may prevent the relevant HPV infections, and presumably, cervical cancer.

\section{Establishment of the Viral Cause}

of Cervical Cancer

Epidemiologic studies are essential in establishing associations between risk factors and cancer, and qualifying the causal nature of the association. Traditionally, these include prevalence surveys in case series, case-control studies, cohort studies, and interventional trials. By the year 2000, the epidemiologic evidence included a large and consistent body of studies indicating beyond any reasonable doubt the existence of strong and specific associations between HPV infection and cervical cancer. The observations are consistent in all countries where investigations have taken place and have satisfied, in biologic terms, the long-term clinical and epidemiologic observations that cervical cancer displays the profile of a sexually transmitted disease (STD).

\section{The Prevalence of HPV DNA in Tissue or Exfoliated Cells from Cervical Cancer}

Case-control studies, case series, and prevalence surveys have unequivocally shown that, with currently available technology, HPV DNA can be recovered from adequate specimens of cervical cancer in $90 \%$ to $100 \%$ of cases. This proportion compares with a prevalence rate of approximately $5 \%$ to $15 \%$ of cervical specimens from women identified as sound epidemiologic control subjects. Moreover, the reasons for lack of detection of HPV DNA in some cervical cancer specimens are being described. For example, in one large investigation, biopsies of over 1000 cases of cervical cancer from 22 countries were examined using the MY09/11 polymerase chain reaction (PCR) system, resulting in a reported HPV DNA prevalence of 93.0\% [1]. Subsequently, the apparently HPV-negative cases were further investigated. These investigations included comparison of the serologic and epidemiologic profiles of the HPV DNA-negative and HPV DNA-positive cases, assessment of the presence of cancer tissue and the quality of the biopsy used for HPV DNA detection by means of a sandwich pathologic assessment, and use of additional technology for HPV DNA testing. In brief, shorter primers in the E7 region, two sets of consensus primers (CPI/II), and the general primers $(\mathrm{GP} 5+/ 6+)$ were used. The final results of the study indicate that the epidemiologic profiles of the two groups were similar ( $i e$, they were both related to the number of sexual partners) and that the vast majority of the apparent HPV-negative cases were in fact HPV-positive. 


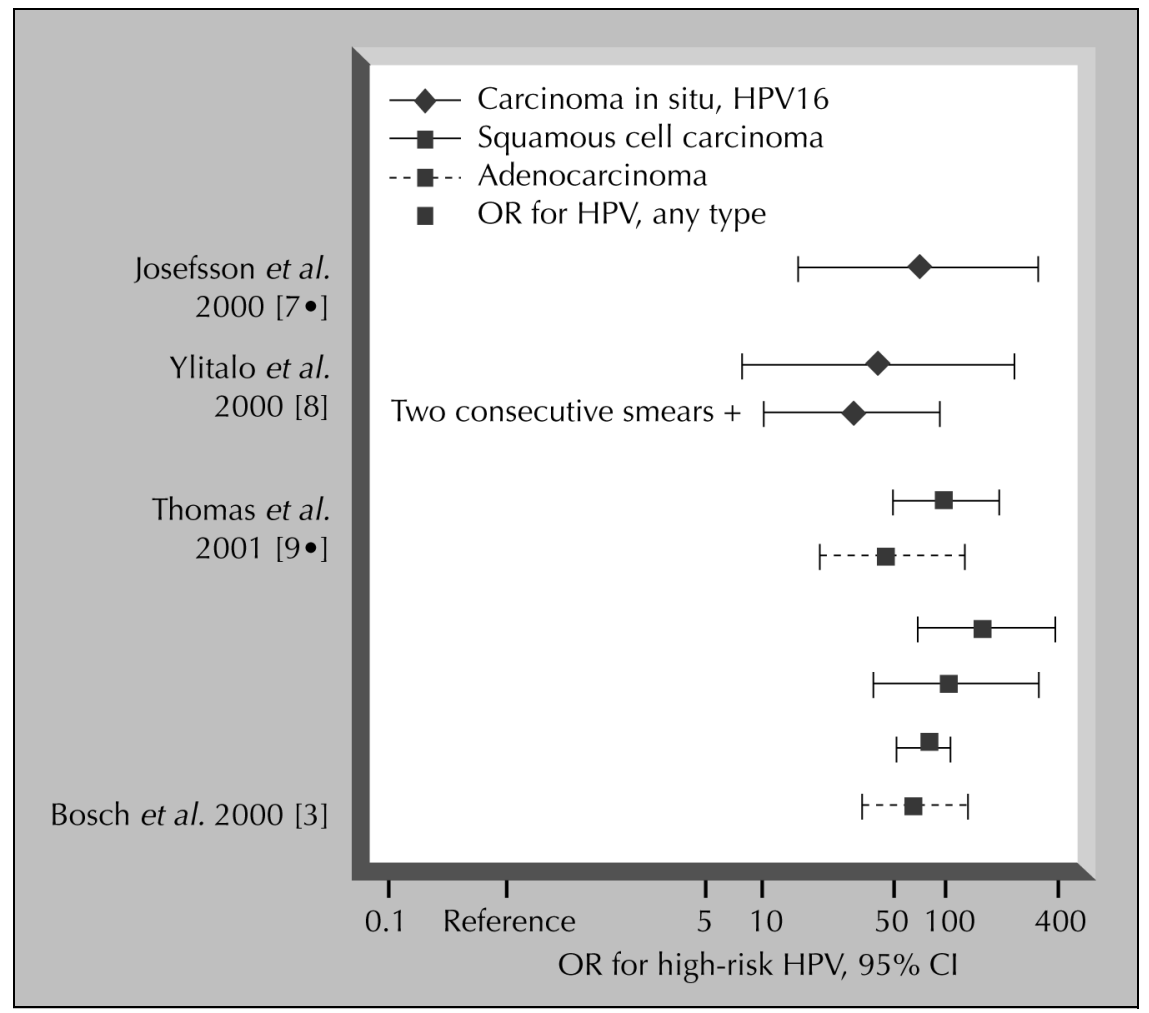

Figure 1. Estimates of the risk of cervical cancer or carcinoma in situ related to the presence of HPV DNA.

Moreover, the HPV types that were newly identified were the common HPV types, with an overrepresentation of HPV18. HPV18 is often integrated into cellular DNA and, in the process, the DNA chain is disrupted, facilitating the lack of recognition by the longer DNA probes. Based on the final results of this study and the considerable body of evidence accumulated from other studies at the time, HPV

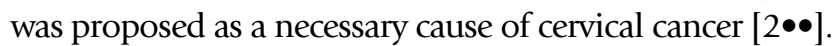

\section{HPV Types}

Of the more than 35 HPV types found in the genital tract, 10 (HPV types 16, 18, 31, 33, 35, 45, 51, 52, 58, and 59) have been adequately evaluated as high-risk types in relation to invasive cervical cancer. For all of these HPV types, risk estimates were greater than 30 (range, 35 to 350), strongly suggesting that these associations are not attributable to chance or to biased study design. HPV16 accounts for $50 \%$ to $70 \%$ of the cervical cancer cases in most countries, followed by HPV18 (10\% to $12 \%$ ) and HPV31 and 45 (4\% to 5\% each). Cervical adenocarcinoma showed a slightly different distribution. The most common types are HPV16 (about 45\%), HPV18 (about 40\%), and HPV45 and 59 ( $4 \%$ to $5 \%$ each). In a series of women without cervical lesions, (corresponding to control subjects in most case-control studies or HPV prevalence surveys from the general population), the distribution of HPV types is much wider. HPV 16 remains the most common type (about 20\%), followed by HPV18 (about 10\%) HPV45 (about 8\%), HPV59 (about 2\%), and smaller proportions of some 30 additional HPV types. Many of these rare types are occasionally found in cases and still convey a high risk of cervical cancer [3].

Interestingly, the geographic variation in the distribution of HPV types has not been fully documented. Some recent studies from areas where little work has been done in the past suggest that additional variability could be expected. For example, high rates of HPV35 and 58 in the general population in Mozambique are now being reported [4]. New technical developments are also describing high frequencies of some rarer HPV types and of multiple HPV infections that were most probably undetected by previous HPV DNA testing systems (Kornegay J, Personal communication).

Finally, studies on HPV variants (variation within HPV types affecting down to one nucleotide of the viral genome) are beginning to reveal that the risk of some HPV16 variants (designated as non-European and nonEuropean-like) may differ from that of the HPV16 European prototype $[5,6]$. The geographic distribution of HPV variants is still being described, and its relevance for HPV testing and for vaccine development is still uncertain.

\section{Case-Control Studies}

Figure 1 displays the key results of some of the most relevant case-control studies concerning HPV and cervical cancer reported in the years 2000 and 2001. The investigators for these published studies have reported odds ratios (OR) for cervical cancer in the range of 50- to 100-fold for HPV DNA. Risk estimates for specific associations like HPV16 and squamous cell cancer or HPV18 and cervical adenocarcinoma 
Table I. Selected examples of the strength of the associations between risk factors and human cancer, with estimates of attributable and protective fractions

\begin{tabular}{|clc|}
\hline Relative risk/odds ratio & Example & Attributable fraction, \% \\
\hline 500 & HPV DNA-I8 and cervical adenocarcinoma in the Philippines & 99 \\
& HPV DNA-I6 and cervical cancer in Costa Rica & 80 \\
I00 & HPV DNA and cervical cancer in Bangkok, Thailand & 90 \\
50 & HBsAg and liver cancer in Taiwan & 60 \\
20 & HBsAg and liver cancer in Greece & 60 \\
10 & HCV and liver cancer in Italy & 40 \\
Baseline reference (I) & Cigarette smoking and lung cancer & 80 \\
0.1 & Smoking cessation before middle age in the UK and lung cancer & Protective fraction, \% \\
0.6 & HBV-vaccinated adults in Korea and liver cancer & 90 \\
0.1 & HBV-vaccinated newborns in Taiwan and liver cancer & 40 \\
\hline HBV-hepatitis B virus; HBsAg-hepatitis B surface antigen; HCV-human papillomavirus. & 90 \\
\hline
\end{tabular}

range between 100 and 900 . The calculation of attributable fractions (AF) from recent studies has consistently indicated proportions greater than $95 \%$ [3]. For practical purposes, the evidence should be interpreted as if all cases of cervical cancer worldwide were related to the persistence of HPV DNA in the cervical cells. As a consequence, it is now recognized that preventive strategies based on the concept of a two-model disease are no longer justified.

Table 1 shows, for comparison, the magnitude of the risk estimates (OR or RR [relative risk]) representing the most frequently observed associations of other viral carcinogens. Table 1 also includes estimates of the protection level achieved under controlled circumstances by vaccination against HBV infection. References to similar calculations of the risk and protection associations between smoking and lung cancer are also shown.

\section{Follow-up Studies}

Follow-up studies (also known as cohort studies) have monitored women with repeated Papanicolaou's (Pap) smears and HPV viral tests from cytologic normalcy to the stage of high-grade squamous intraepithelial lesion (HSIL), at which point intervention is mandatory. These studies provide useful information on the natural history of HPV infection, its risk factors for acquisition, and its persistence. In addition, they successfully document the fact that viral exposure precedes the development of cervical neoplasm.

Repeated sampling of women followed for viral persistence and cervical abnormalities has shown that the spontaneous median duration of infection is around 8 months for high-risk HPV types, compared with 4 to 5 months for low-risk HPV types. HPV16, the most common high-risk type, showed the longer duration, with average estimates of 13.5 months in a high-risk population in Brazil [10]. The results were remarkably similar in a student population in the United States [11] and in a low-risk population in Spain (de Sanjosé S et al., Personal communication). The self-limiting course of most HPV infections is consistent with the cross-sectional profile displayed in Figure 2, taken from the population in The Netherlands $[14,15]$. The profiles are very similar to those from most other populations worldwide; however, in some high-risk populations, a bimodal distribution has been observed with a second increase in the prevalence of HPV DNA in the older age groups $[12,13]$. The significance of this second peak in viral detection has not been elucidated.

Follow-up studies of women without cervical abnormalities indicate that the continuous presence of HPV DNA is necessary for the development, maintenance, and progression of cervical intraepithelial neoplasia (CiN) $[11,16]$. A substantial proportion (ie, $6 \%$ to $10 \%$ ) of women with high-risk HPV DNA who are cytologically normal at recruitment will develop CiN3 within the subsequent 4-year interval $[17,18]$. Conversely, women found to be HPV DNA negative and cytologically identified as having atypical squamous cells of undetermined significance (ASCUS), borderline disease, or mild dysplasia are unlikely to develop CiN3 during a follow-up period of at last 2 years. Women found to be positive for low-risk HPV rarely become persistent carriers, and the probability of their progression to HSIL is extremely low $[19,20]$. The negative predictive value of the combination of a negative HPV test and a normal cytology probably extends well over 5 years, and this offers the most attractive perspective in rationalizing screening programs. Clearance of high-risk HPV seems to be associated with regression of CiN lesions $[20,21]$. Finally, persistence of HPV DNA detection after treatment of preinvasive neoplasia is an accurate predictor of relapse,

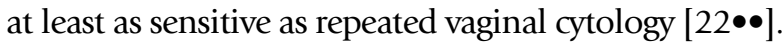

\section{Retrospective Cohorts}

A particularly interesting approach to conducting followup studies of invasive cancer (as opposed to studies of CiN3) is provided by studies initiated in 1969 with followup in 1995 that assembled and stored banks of biologic specimens from healthy individuals. Linkage studies can 


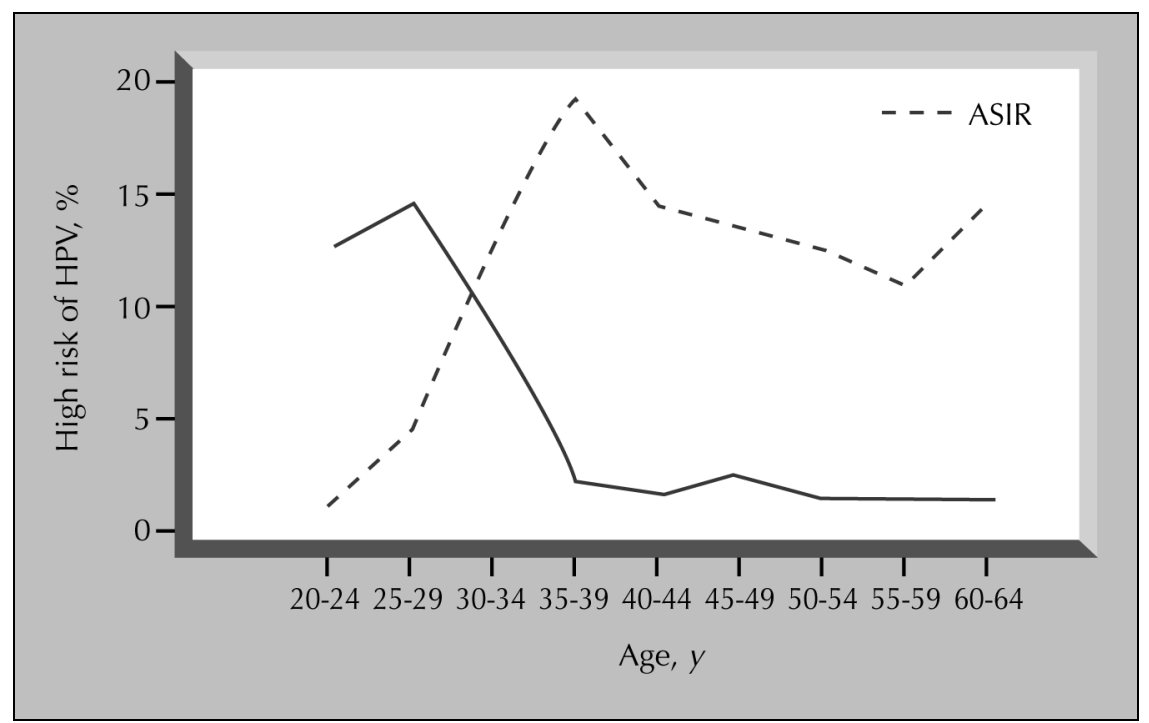

Figure 2. Age-specific prevalence of high-risk HPV DNA in 3700 women entering a screening program and age-specific incidence rate of cervical cancer in The Netherlands.

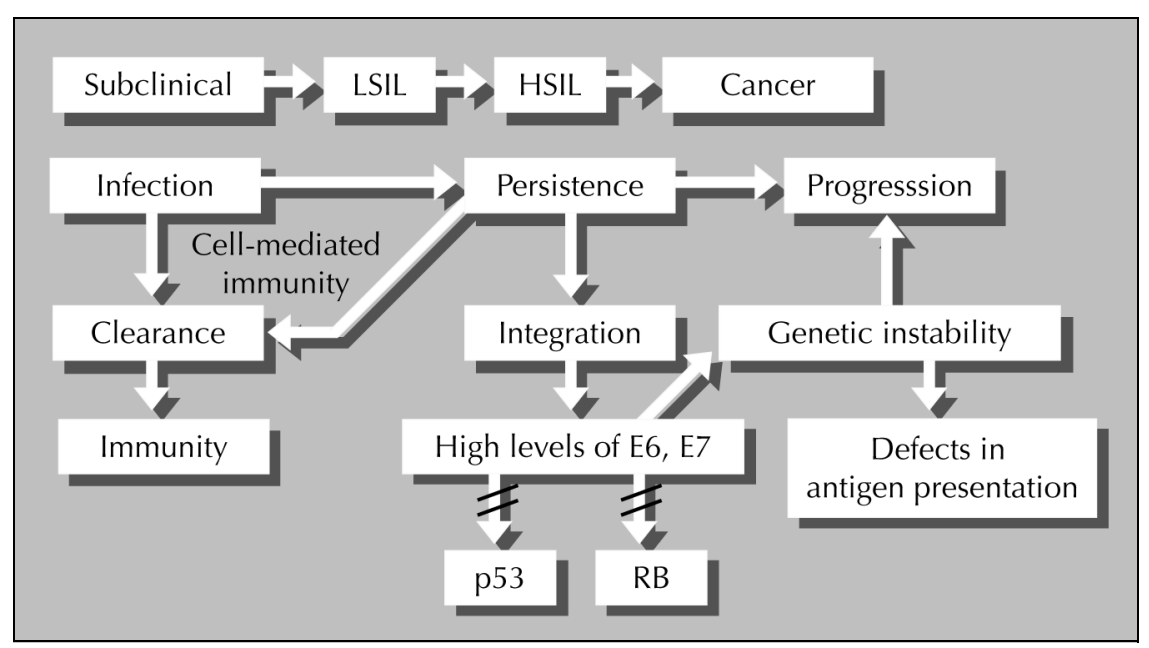

Figure 3. Mechanisms of HPV carcinogenesis.

then identify cases of cervical cancer (or any other condition) that have occurred in the intervening period, and the original specimens can be analyzed for the presence of HPV biomarkers. HPV DNA prevalence, for example, can be compared with the corresponding prevalence in specimens from epidemiologically sound individuals from the same cohort who did not develop the condition under otherwise equivalent exposure records. These studies have documented the presence of HPV DNA or HPV antibodies years before the development of cervical cancer $[23,24]$.

\section{Biologic Mechanisms of Viral Carcinogenesis}

Figure 3 shows some of the major components of the transition from HPV infection to cervical cancer. Whereas transient infections are largely subclinical, progression is closely related to persistent presence of viral DNA. This process frequently goes along with viral disruption in the early (E) E1/E2 regions of the viral genome and integration into the cellular DNA. E2 disruption releases the viral promoters of E6 and E7 and increases expression of these transforming genes. The E6 and E7 viral proteins are capa- ble of selectively degrading the proteins of the $p 53$ gene and of the retinoblastoma $(p R B)$ gene, leading to inactivation of two important cellular negative regulatory proteins.

Several biologic and molecular characteristics that support the role of HPV in the induction of cervical cancer were recently outlined [25]. Accordingly, the causal nature of this association is indicated by the following features: 1) regular presence of HPV DNA in the respective tumor biopsy specimen; 2) demonstration of viral oncogene expression (EG and $E 7$ ) in tumor material; 3 ) transforming properties of these genes (EG and $E 7) ; 4$ ) requirement of $E 6$ and $E 7$ expression for maintaining the malignant phenotype of cervical carcinoma cell lines; 5) interaction of viral oncoproteins with growth-regulating host-cell proteins; and 6) epidemiologic studies pointing at these HPV infections as the major risk factors for cervical cancer development. The criteria outlined by zur Hausen [25] have been evaluated in relation to HPV on a number of occasions, and excellent reviews are available [26].

In reviewing work on the molecular genetics of cervical carcinoma, Lazo [27] indicated three different mechanisms of cancer induction: two HPV-related mechanisms, which 


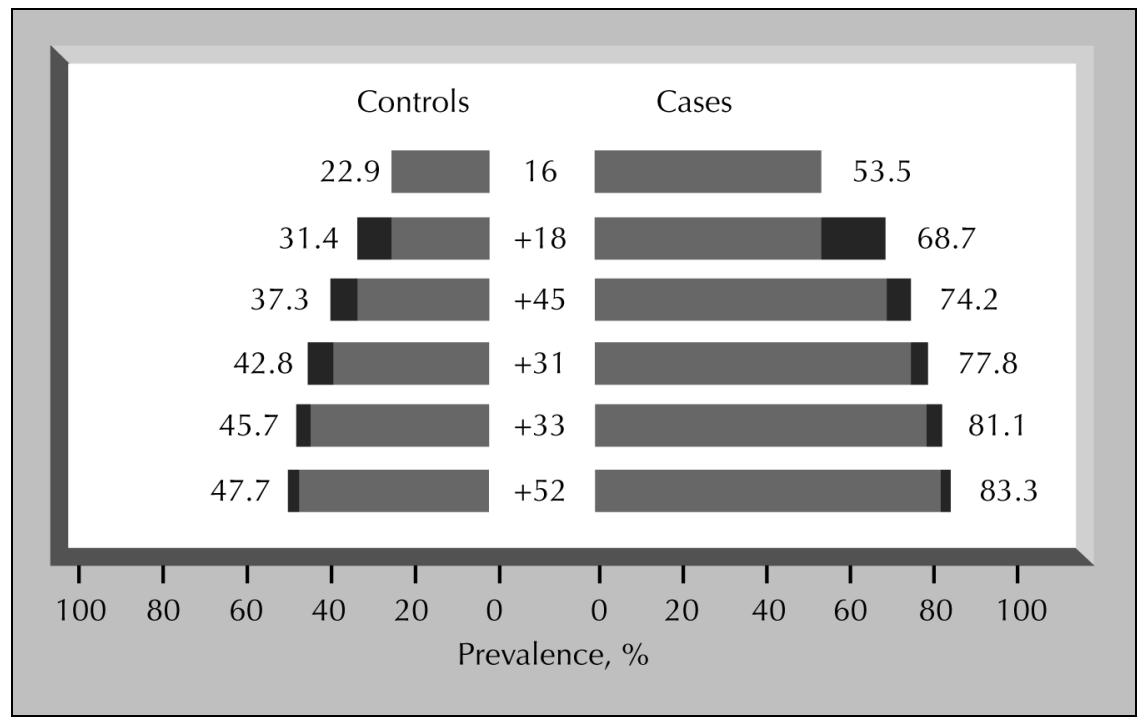

Figure 4. Cumulative prevalence by HPV type.

are the effects of E6 and E7 on host regulatory proteins, and a third effect of viral integration and its specific impact in relation to the integration sites. The third mechanism, which may or may not be related to HPV, is the accumulation of cellular genetic damage needed for tumor development. The existence of this mechanism is strongly suggested by the observation of recurrent loss of heterozygosity and by recurrent amplification in a large proportion of cervical carcinomas. The role of an unidentified tumor suppressor gene is also suggested by experiments showing that the tumorigenicity of He-La cells could be suppressed by fusion with normal fibroblasts or keratinocytes.

\section{Preventive and Clinical Implications} Vaccination against HPV

Given the strong relationship between HPV infection and cervical cancer, prevention of persistent HPV infection seems to be a desirable target and perhaps the only realistic option for developing countries. The profile of HPV prevalence across age groups (Fig. 2), and the limited duration of most HPV infection estimated by cohort studies, is generally interpreted as the manifestation of a fairly active immune response that includes antibody production and cell-mediated immune responses. A typical HPV infection of the cervical tissue calls for viral replication in the suprabasal, superficial squamous epithelia without a viremic phase. The process occurs entirely in cells that are undergoing terminal differentiation and largely escapes immunologic surveillance mechanisms.

Individuals who have successfully cleared a specific type of HPV infection are protected against reinfection with the same HPV type, but evidence indicates that crossprotection against closely related HPV types does not occur or occurs at very low levels [28]. Prophylactic vaccines against HPV, currently under development and evaluation, include products that aim to prevent new infection. Therapeutic vaccines aim to resolve infection with or without early cytologic lesions, and combined vaccines target both effects. At present, over 30 ongoing trials are using both the prophylactic and the therapeutic vaccines.

Because cross-protection is limited, one of the central issues in exploring products destined for widespread use is the number of viral types that are to be included. Figure 4 shows the cumulative prevalence of HPV types in over 2000 cases of invasive cervical cancer from over 25 countries. This series corresponds with the cases entered in the International Agency for Research on Cancer (IARC) register of studies on HPV and cervical cancer. It appears that four HPV types-16, 18, 45, and 31explain close to $80 \%$ of the types of infection involved in cervical cancer worldwide. The variability is somewhat less marked for cervical adenocarcinoma, in which HPV types $16,18,45$, and 59 account for $93 \%$ of the types found in cases. Finally, the distribution of HPV types is greater in samples of women from the general population of the same country, where the same four types account for approximately $45 \%$ of the distribution.

Results from vaccination trials in animal models (dogs, cattle, and rabbits) are encouraging. Experiments with virallike particles (VLP) based on the major structural viral protein L1, or directly with the L1 gene, are capable of inducing antibody responses and high protection levels against viral challenges. Phase I and II clinical trials with prophylactic vaccines have been completed in humans, and large phase III trials are in advanced phases of planning.

Table 2 provides an estimate of the likely impact of a vaccine as a function of the number of HPV types included in the product, the vaccine efficacy ratio, and the level of coverage of the target population. As in other vaccination schemes, these estimates indicate that population coverage is an important determinant, probably with greater impact on the global efficiency of the system than the number of HPV types included in the vaccine.

Therapeutic vaccines may be interesting alternatives in populations in which a large proportion of young adult 
Table 2. Theoretical reduction in cervical cancer incidence under different vaccination products and target population coverage

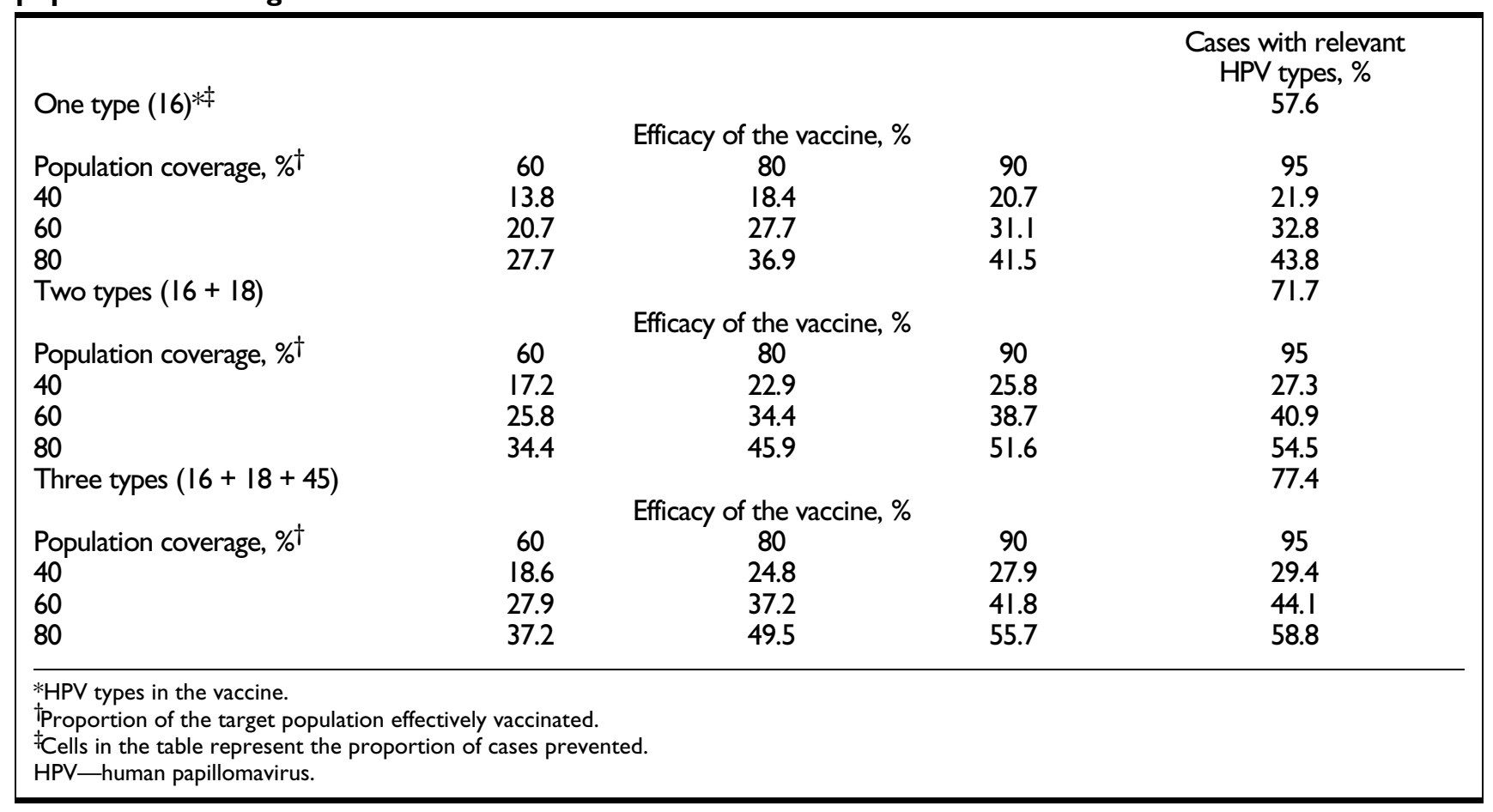

women are already permanent carriers of HPV DNA. These products incorporate modified fragments of the $E G$ and/or $E 7$ genes, the viral products consistently expressed in persistent infection and in cervical cancer. Chimeric VLPs have been shown to induce antigen-specific protection in mice from challenge with E7-expressing tumor cells [29].

\section{Screening with HPV tests}

Cervical cytology has played an important role in screening and clinical management of cervical lesions. A recognized barrier, however, is the limited sensitivity and specificity of cervical cytology in most settings, leading to false-positive and false-negative reports. One meta-analysis, for example, indicated $98 \%$ specificity and $50 \%$ sensitivity for cervical cytology [30•]. In a number of countries, cytologic screening has proven difficult to implement and sustain, and under suboptimal quality control these programs tend to offer very low cost-benefit results. It has been suggested that screening based on HPV DNA testing may prove easier to implement and sustain. Considerable efforts are currently devoted to the testing of this hypothesis.

Because the risk of developing cervical cancer and the prognosis are similar for the different types of high-risk HPV, it is generally accepted that test formats that detect the known high-risk HPV types in a cocktail mix are suitable for screening. One such test, the Hybrid Capture 2 (HC2; Diagene, Gaithersburg, MD) is commercially available and progressively introduced in clinical practice. Individual typing remains necessary in research settings and for studies evaluating therapeutic or preventive type-specific $H P V$ vaccines [31,32].

Ideally, HPV screening tests should detect all CIN3/ HSIL and cervical cancer. Both HC2 and GP5+/6+ PCR electroimmunoassay have at least equal sensitivity for CIN3 and cervical cancer, and in most studies results are significantly better than with cervical cytology $[16,19,33,34]$. The specificity of HPV tests is dependent on age. In the young age groups, the specificity of HPV testing is lower than that of cytology, whereas in individuals aged 35 years or more (again country-dependent), the specificity of both tests is similar. Recent studies in which HC2 and GP5+/6+ were used showed that both tests have a high negative predictive value for CIN and cervical cancer $[16,22 \bullet \bullet, 33,34,35 \bullet \bullet]$. In combination, women with both normal cytology and absence of HPV DNA have an extremely low risk of developing cervical cancer in 10 or more years. Major gains in effectiveness and cost reduction are to be expected from increasing screening intervals and reducing the total number of visits requested per lifetime in most cytologic screening programs.

Table 3 summarizes the results of a number of studies that have evaluated the performance of HPV DNA as a screening test. Several of these studies have been conducted in low-middle income countries, settings in which cytologic screening has proven to be difficult to sustain. Some of these evaluations may overestimate the sensitivity of the HPV DNA tests as a consequence of detection bias. This bias occurs whenever a second screening test is added to improve disease detection. Some of the gains in sensitivity 
Table 3. Performance of HPV tests for detection of HSIL and cancer in selected studies that mimic population-based screening conditions

\begin{tabular}{|c|c|c|c|c|c|c|}
\hline \multirow[t]{2}{*}{ Study } & \multirow[t]{2}{*}{ Country } & \multirow[t]{2}{*}{ Test } & \multicolumn{2}{|c|}{ HPV DNA } & \multicolumn{2}{|c|}{ Cytology } \\
\hline & & & Sensitivity, \% & Specificity, \% & Sensitivity, \% & Specificity, \% \\
\hline Kuhn et al. [36] & South Africa & $\mathrm{HCl}$ & 73 & 88 & 78 & 97 \\
\hline Schiffman et al. [37] & Costa Rica & $\mathrm{HC2}$ & 88 & 89 & 78 & 94 \\
\hline Belinson et al. [38] & China & $\mathrm{HC2}$ & 98 & 85 & 94 & 78 \\
\hline Womack et al. [39] & Zimbabwe & $\mathrm{HC2}$ & 81 & 62 & 44 & 91 \\
\hline Ratnam et al. [40] & Newfoundland & $\mathrm{HC2}$ & 68 & 91 & 27 & 96 \\
\hline Schneider et al. [4I] & Germany & PCR & 89 & 94 & 20 & 99 \\
\hline Blumenthal et al. [42] & Zimbabwe & $\mathrm{HC2}$ & 80 & 61 & 44 & 91 \\
\hline & & $\mathrm{HC} 2 \mathrm{ss}$ & 66 & 82 & - & - \\
\hline Wright et al. [43] & South Africa & $\mathrm{HC} 2$ & 84 & 85 & 61 & 97 \\
\hline Cuzick et al. [44] & UK & $\mathrm{HC}+\mathrm{PCR}$ & 95 & 95 & 79 & 99 \\
\hline
\end{tabular}

(the additional cases identified) occur by chance alone, irrespective of the validity of the second assay. However, when corrections for the chance gain in sensitivity are based on the published results, the general scenario remains quite consistent (Franco E, Personal communication). Recent results from South Africa suggest that direct visual inspection (DVI) or HPV DNA tests may offer attractive alternatives to cytologic screening in populations with limited resources and access to a medium level of technologic sophistication [45].

Estimates of the validity of the new HPV tests may also be affected by the concurrent prevalence of HIV infection. It is now known that HIV-positive women have a higher prevalence of HPV DNA, even in the absence of cytologic abnormalities, than do otherwise comparable groups of HIV-negative women. As a consequence, lower specificity is expected from studies in populations with high HIV infection rates, as shown in Table $3[39,42]$.

HPV testing in the triage of minimal cervical abnormalities

One of the first applications of HPV testing in clinical practice was the secondary triage of women referred for colposcopy because of an abnormal Pap smear. In the United States, adoption of the Bethesda system (TBS) for cervical cytology dramatically increased the proportion of Pap smears with cytologic abnormalities that merited clinical attention [46]. The new terminology increased the overall proportion of low-grade lesions by combining the original mild dysplasia category with cytologic abnormalities consistent with koilocytotic atypias into the low-grade intraepithelial lesion (LSIL) designation. It also created the new ASCUS category for borderline and uncertain abnormalities. ASCUS and LSIL decreased, and smears with equivocal or minor-grade abnormalities represent approximately $4 \%$ to $7 \%$ of all Pap smears in the United States, a considerable workload for the purposes of clinical decision making. Whereas consensus holds that women with HSIL on cytology need immediate referral for colposcopy, there is uncer- tainty about management options for ASCUS and LSIL. International guidelines conservatively propose frequent (every 6 months) cytologic follow-up for the latter patients. However, concerns about malpractice litigation have led to a more aggressive approach in some countries. In practical terms, these attitudes generate a substantial burden of overdiagnosis and overtreatment of conditions that would otherwise regress spontaneously in a significant proportion of patients ( $50 \%$ or more). The use of ancillary tests, notably HPV DNA, for the triage of such patients was thus rapidly proposed and evaluated.

In countries where TBS has not been fully adopted or where cytology programs are not developed, the results from triage studies based on TBS classification have uncertain application. Studies that evaluate local expertise and test (cytology) reproducibility are an important part of the process of adopting the new technology. The best evidence on the role of HPV testing as an alternative to repeated cytology in the presence of an ambiguous abnormal cytology was provided by the Kaiser Permanente study in 1999

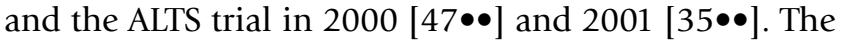
Kaiser Permanente study used concomitant testing focused exclusively on ASCUS as the presumptive diagnosis of the referral smear. Among a cervical cancer screening population of 46,000 women participating in the health maintenance program, 995 ASCUS cases were identified. HPV testing by HC2 of the residual fluid collected for liquidbased cytology was compared with repeat Pap cytology (at a repeated ASCUS threshold) based on the ability to identify women with HSIL on histology. The triage algorithm considered a second cycle of reflex HPV testing on persistent ASCUS smears after 6 months. The proportion of women who would have been referred for colposcopy was comparable in the two approaches: $40 \%$ for HPV and 39\% for repeat Pap smear. The sensitivity to detect HSIL or cancer was $89 \%$ for HPV and 76\% for Pap smear, with equivalent specificity for both tests (64\%).

The ALTS trial, coordinated by the National Cancer Institute (NCI) of the US National Institutes of Health, is a 
randomized clinical trial designed to determine the optimal management plan separately for LSIL and ASCUS cytologic abnormalities. About 600 women with LSIL and approximately 3500 women recently diagnosed with ASCUS were randomly assigned to one of three arms: 1 ) immediate colposcopy; 2) referral to colposcopy if cytology at enrollment (or at any of the follow-up cytologies) showed HSIL or worse lesions, and subsequently to HPV testing (HC2); or 3) HPV (HC2) triage, with referral for colposcopy if the HPV test at enrollment was positive or missing or if any cytology result was positive for HSIL.

In the LSIL component of the ALTS trial, the HPV triage arm was terminated prematurely because of the interim observation of a very high rate (83\%) of oncogenic HPV positivity. Such high HPV prevalence in LSIL is rarely reproduced in other studies, reflecting variability in the diagnosis of LSIL. The authors of the ALTS trial concluded that, in the US setting, there would be limited value in using HPV

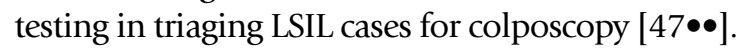

In contrast, the ASCUS component of the trial reinforced the findings of the Kaiser Permanente study. HPV testing yielded $96 \%$ sensitivity in detection of both CiN2+ and CiN3+ histologically confirmed lesions, whereas repeat Pap smear at the lowest threshold of ASCUS produced a significantly lower sensitivity of $85 \%$ for both definitions of lesion severity. The sensitivity of a repeated cytology at a threshold level of CiN3 + was as low as $44 \%$. The proportions of women who needed referral for colposcopy due to positive test results were $56 \%$ and $59 \%$, respectively. The investigators concluded that HPV testing was a viable option in the triage and management of ASCUS smears [35••]. Issues of cost effectiveness have been partially addressed so far by these two studies, but preliminary analyses indicate that HPV testing is superior to aggressive management by immediate colposcopy or conservative cytologic follow-up.

\section{Opportunities for self sampling in screening and triage}

Several studies have now evaluated the possibility of using self-collected vaginal samples for HPV DNA testing $[43,48]$. Although, in general, the results have been promising, reduced sensitivity for detection of HSIL and cancer has been observed in those studies in which samples were collected by the patients themselves compared with those in which samples were collected by clinicians. In a study of 200 women enrolled from colposcopy clinics, the sensitivity of HC2 for detection of HSIL was $98 \%$ when the test was performed on clinician-collected samples and $86 \%$ when performed on self-collected samples [48]. In a screening setting, Wright $e t$ al. [43] found $84 \%$ sensitivity of HC2 for HSIL and cancer with clinician-collected samples, compared with $69 \%$ for self-collected samples. In countries where trained professionals may be lacking and other cultural barriers prevent access to screening, self sampling may prove to be an important development for the prevention of cervical cancer.

\section{Conclusions}

At the current level of understanding, the causal role of persistent HPV infection in the development of cervical cancer and its precursors has been proven beyond any reasonable doubt. Screening strategies based on the judicious use of HPV testing combined with cytology have the potential to increase accuracy and efficiency over and above any previously tried screening strategy for cervical cancer. When HPV vaccines are available, vaccination against HPV may prove to be the most efficient preventive strategy for most parts of the world.

\section{References and Recommended Reading}

Papers of particular interest, published recently, have been highlighted as:

- Of importance

- Of major importance

1. Bosch FX, Manos MM, Muñoz N, et al., for the International Biological Study on Cervical Cancer (IBSCC) Study Group: Prevalence of human papillomavirus cervical cancer: a worldwide perspective. J Natl Cancer Inst 1995, 87:796-802.

2.• Walboomers JM, Jacobs MV, Manos MM, et al.: Human papillomavirus is a necessary cause of invasive cervical cancer worldwide. J Pathol 1999, 189:12-9.

Along with Bosch et al. [1], this paper reports on an exhaustive investigation of the presence of viral DNA in over 1000 cervical cancer cases from 22 countries. The final reported prevalence of $99.7 \%$ generated the claim that HPV is in fact a necessary cause of cervical cancer.

3. Bosch FX, Rohan T, Schneider A, et al.: Papillomavirus research update: highlights of the Barcelona HPV 2000 International Papillomavirus Conference. J Clin Pathol 2001, 54:163-175.

4. Castellsagué X, Menéndez C, Loscertales M-P, et al.: Human papillomavirus genotypes in rural Mozambique. Lancet 2001, 358:1429.

5. Xi LF, Koutsky LA, Galloway DA, et al.: Genomic variation of human papillomavirus type 16 and risk for high grade cervical intraepithelial neoplasia. J Natl Cancer Inst 1997, 89:796-802.

6. Hildesheim A, Schiffman M, Bromley C, et al:: Human papillomavirus type 16 variants and risk of cervical cancer. J Natl Cancer Inst 2001, 93:315-318.

7. Josefsson AM, Agnetha M, Magnusson PKE, et al:: Viral load of human papilloma virus 16 as a determinant for development of cervical carcinoma in situ: a nested case-control study. Lancet 2000, 355:2189-2193.

This paper represents an evaluation of HPV viral load as an additional test to predict progression in screening specimens at stages in which cytology is uninformative. It is one of the first studies to include quantitative PCR to generate estimates of the viral load.

8. Ylitalo N, Josefsson A, Melbye $M$, et al.: A prospective study showing long-term infection with human papillomavirus 16 before the development of cervical carcinoma in situ. Cancer Res 2000, 60:6027-6032.

9. Thomas DB, Ray RM, Koetsawang A, et al.: Human papillomaviruses and cervical cancer in Bangkok. I. Risk factors for invasive cervical carcinomas with human papillomavirus types 16 and 18 DNA. Am J Epidemiol 2001, 153:723-731.

This is the most recent report on a large case-control study on HPV and cervical cancer. It confirms the key findings of the previous studies by Bosch et al. [3] and Herrero et al. [12] on the extremely high relative risk estimates for the presence of HPV DNA as a determinant of invasive cervical cancer or preinvasive cervical neoplasm.

10. Franco EL, Villa LL, Sobrinho JP, et al.: Epidemiology of acquisition and clearance of cervical human papillomavirus infection in women from a high-risk area for cervical cancer. $J$ Infect Dis 1999, 180:1415-1423.

11. Ho GYF, Biermal R, Beardsley L, et al.: RD: Natural history of cervicovaginal papillomavirus infection in young women. $N$ Engl J Med 1998, 338:423-428. 
12. Herrero R, Hildesheim A, Bratti C, et al:: Population-based study of human papillomavirus infection and cervical neoplasia in rural Costa Rica. J Natl Cancer Inst 2000, 92:464-474.

13. Lazcano-Ponce E, Herrero R, Muñoz N, et al.: Epidemiology of HPV infection among mexican women with normal cervical cytology. Int J Cancer 2001, 91:1-9.

14. Jacobs MV, Walboomers JMM, Snijders PJF, et al.: Distribution of 37 mucosotropic HPV types in women with cytologically normal cervical smears: the age-related patterns for high-risk and low-risk types. Int J Cancer 2000, 87:221-227.

15. Parkin DM, Whelan SL, Ferlay J, et al.: Cancer Incidence in Five Continents, vol 7. Lyon: IARC Scientific Publications; 1997.

16. Nobbenhuis MAE, Walboomers JMM, Helmerhorst TJM, et al. Relation of human papillomavirus status to cervical lesions and consequences for cervical-cancer screening: a prospective study. Lancet 1999, 354:20-25.

17. Rozendaal L, Walboomers JM, van der Linden JC, et al:: PCRbased high-risk HPV test in cervical cancer screening gives objective risk assessment of women with cytomorphologically normal cervical smears. Int J Cancer 1996, 68:766-769.

18. Rozendaal L, Westerga J, van der Linden JC, et al.: PCR based high risk HPV testing is superior to neural network based screening for predicting incident CIN III in women with normal cytology and borderline changes. J Clin Pathol 2000, 53:606-611.

19. Manos MM, Kinney WK, Hurley LB, et al.: Identifying women with cervical neoplasia: using human papillomavirus DNA testing for equivocal Papanicolaou results. JAMA 1999, 281:1605-1610.

20. Zielinski GD, Snijders PJF, Rozendaal L, et al.: HPV presence precedes abnormal cytology in women developing cervical cancer and signals false negative smears. Br J Cancer 2001, 85:398-404

21. Nobbenhuis MA, Helmerhorst TJ, van den Brule AJ, et al.: Cytological regression and clearance of high-risk human papillomavirus in women with abnormal cervical smear. Lancet 2001, 358:1782-1783.

$22 . \bullet$ Nobbenhuis MA, Meijer CJ, van den Brule AJ, et al.: Addition of high-risk HPV testing improves the current guidelines on follow-up after treatment for cervical intraepithelial neoplasia. Br J Cancer 2001, 84:796-801.

Description of the ability of the HPV tests after conization to predict CiN2 or 3 at 24 months following treatment. The sensitivity at 6 months of an HPV test was $90 \%$, compared with cytology at $62 \%$. The negative predictive value of an HPV result was 99\%. The study was based on 184 women with a 24-month follow-up and $29 \mathrm{CiN} 2$ or 3 events.

23. Wallin K-L, Wiklund F, Angström T, et al.: Type-specific persistence of human papillomavirus DNA before the development of invasive cervical cancer. N Engl J Med 1999, 341:1633-1638.

24. Dillner J, Lehtinen M, Bjorge T, et al.: Prospective seroepidemiologic study of human papillomavirus infection as a risk factor for invasive cervical cancer. J Natl Cancer Inst 1997, 89:1293-1299.

25. zur Hausen H: Papillomavirus causing cancer: evasion from host-cell control in early events in carcinogenesis. $J$ Natl Cancer Inst 2000, 92:690-698.

26. Shah KV, Howley PM: Papillomaviruses. In Field's Virology. Edited by Knipe DM, Howley PM. Philadelphia: LippincottRaven; 1996:2077-2109.

27. Lazo PA: The molecular genetics of cervical carcinoma. $\mathrm{Br} \mathrm{J}$ Cancer 1999, 80:2008-2018.

28. Thomas KK, Hughes JP, Kuypers JM, et al.: Concurrent and sequential acquisition of different genital human papillomavirus types. J Infect Dis 2000, 182:1097-102.

29. Schiller JT, Lowy DR: Papillomavirus-like particle vaccines. J Natl Cancer Inst Monogr 2000, 28:50-54.
30. Nanda K, McCrory DC, Myers ER, et al.: Accuracy of the Papanicolau test in screening for and follow-up of cervical cytologic abnormalities: a systematic review. Ann Intern Med 2000, 132:810-819.

This is an updated meta-analysis of the relative value of cytology as a screening test. The authors report sensitivity of close to $50 \%$ and specificity of $98 \%$.

31. Kleter B, van Doorn LJ, ter Schegget J, et al.: Novel short-fragment PCR assay for highly sensitive broad-spectrum detection of anogenital human papillomaviruses. Am J Pathol, 1998, 153:1731-1739.

32. Gravitt PE, Peyton CL, Alessi TQ, et al.: Improved amplification of genital human papillomaviruses. J Clin Microbiol 2000, 38:357-361.

33. Hutchinson ML, Zahniser DJ, Sherman et al.: Utility of liquidbased cytology for cervical carcinoma screening : results of a population-based study conducted in a region of Costa Rica with a high incidence of cervical carcinoma. Cancer 1999, 87:48-55.

34. Clavel C, Measure M, Levert $M$, et al.: Human papillomavirus detection by the hybrid capture II assay : a reliable test to select women with normal cervical smears at risk for developing cervical lesions. Diagn Mol Pathol 2000, 9:145-150.

$35 . \bullet$ Solomon D, Schiffman M, Tarone R: Comparison of three management strategies for patients with atypical squamous cells of undetermined significance: baseline results from a randomized trial. J Natl Cancer Inst 2001, 93:293-299.

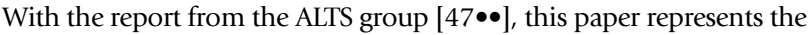
first large randomized trial to evaluate clinical management of cytology results diagnosed as ASCUS or LSIL. The authors confirm the value of HPV testing over repeated cytology in clarifying the prognosis of an ambiguous expert cytology, classified according to the Bethesda system.

36. Kuhn L, Denny L, Pollack A, et al.: Human papillomavirus DNA testing for cervical cancer screening in low- resource settings. J Natl Cancer Inst 2000, 92:818-825.

37. Schiffman MH, Herrero R, Hildesheim A, et al.: HPV DNA testing in cervical cancer screening. JAMA 2000, 283:87-93.

38. Belinson J, Qiao Y, Pretorius R, et al.: Prevalence of cervical cancer and feasibility of screening in rural China: a pilot study for the Shanxi Province Cervical Cancer Screening Study. Int J Gynecol Cancer 1999, 9:411-417.

39. Womack SD, Chirenje ZM, Gaffikin L, et al.: HPV-based cervical cancer screening in a population at high risk for HIV infection. Int J Cancer 2000, 85:206-210.

40. Ratnam S, Franco EL, Ferenczy A: Human papillomavirus testing for primary screening of cervical cancer precursors. Cancer Epidemiol Biomarkers Prevent 2000, 9:945-951.

41. Schneider A, Hoyer H, Lotz B, et al.: Screening for high-grade cervical intra-epithelial neoplasia and cancer by testing for high-risk HPV, routine cytology or colposcopy. Int J Cancer 2000, 89:529-534.

42. Blumenthal PD, Gaffikin L, Chirenje ZM, et al: : Adjunctive testing for cervical cancer in low resource settings with visual inspection, HPV, and the Pap smear. Int J Gynecol Obstet 2001, 72:47-53.

43. Wright TC Jr, Denny L, Kuhn L, et al.: HPV DNA testing of selfcollected vaginal samples compared with cytologic screening to detect cervical cancer. JAMA 2000, 283:81-86.

44. Cuzick J, Beverley E, Ho L, Terry G, Sapper H, Mielzynska I, Lorinzc A, Chan W-K, Krausz T, Soutter P: HPV testing in primary screening of older women. Br J Cancer 1999, 81(3):554-58.

45. Goldie SJ, Kuhn L, Denny L, et al.: Policy Analysis of cervical cancer screening strategies in low-resource settings: clinical benefits and cost-effectiveness. JAMA 2001, 285:3107-3115.

46. Solomon D: The 1988 Bethesda system for reporting cervical/ vaginal cytologic diagnoses. Developed and approved at the National Cancer Institute Workshop, Bethesda, Maryland, USA, December, 12-13, 1988. J Clin Cytol Cytopathol 1989, 33:567-574. 
47.• The Atypical Squamous Cell of Undetermined Significance/ Low-grade squamous Intraepithelial Lesions Triage Study (ALTS) Group: Human papillomavirus testing for triage of women with cytologic evidence of low-grade squamous intraepithelial lesions: baseline data from a randomized trial. J Natl Cancer Inst 2000, 92:397-402.

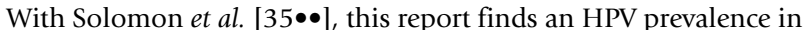
LSIL of $83 \%$, suggesting that, under these diagnostic conditions, HPV testing would not be of value as a triage for coloscopy criteria.
48. Sellors JW, Mahony JB, Kaczorowski J, et al., for the Survey of HPV in Ontario Women (SHOW): Group prevalence and predictors of human papillomavirus infection in women in Ontario, Canada. CMAJ 2000, 163:503-508. 